\title{
A Mathematical Model to Solve the Burgers-Huxley Equation by using New Homotopy Perturbation Method
}

\author{
Dinesh Kumar Maurya \\ Department of Mathematics, \\ K. R. Mangalam University, Gurgaon -122103, Haryana, India \\ E-mail: dkmupc@gmail.com \\ Ravendra Singh \\ Department of Mathematics, \\ K. R. Mangalam University, Gurgaon-122103, Haryana, India \\ E-mail: ravendraibs@gmail.com \\ Yogendra Kumar Rajoria \\ Department of Mathematics, \\ K. R. Mangalam University, Gurgaon-122103, Haryana, India \\ Corresponding author: yogendrarajo@gmail.com
}

(Received April 27, 2019; Accepted August 28, 2019)

\begin{abstract}
A semi-analytical method has been planned for the precise solution of the differential equation established on the New Homotopy Perturbation Method (NHPM), and to develop a generalized Burger-Huxley (BH) equation, in this paper. By employing NHPM, two case studies show the precise solution of the BH equation. It is shown that the NHPM is yield solution is convergent from with the easy computability term; NHPM is an effective and easy tool for cracking many real world difficulties. The three-dimension and two dimension graphical solutions of the $\mathrm{BH}$ equations are also provided to validate the mathematical models. MATLAB software is used to calculate the series obtained from HPM.
\end{abstract}

Keywords- Burgers-Huxley equation, New homotopy perturbation method, MATLAB software.

\section{Introduction}

Most incidents in the real world play a significant character in the study of nonlinear equations that pass through many studies. The BH equations come from the mathematical modelling of various systematic events. Generalized $\mathrm{BH}$ equation demonstrates a model for depicting the collaboration amid convection impacts, reaction mechanisms and diffusion transports. Satsuma (1887) investigated the generalised BH equation. The non-linear reaction-diffusion equations play a very important role to solve the application extended over the pharmaceuticals, porous soil, semiconductors doping process, biology, chemistry, atmospheric science and many other arenas. This approximate closed-form solution is often beneficial for engineers and others to describe reaction-diffusion equations type of linear and non-linear. Mostly the previous study of nonlinear reaction-diffusion equations did not an analytical solution. Recently, we considered the results of everyday problem of non-linear reaction-diffusion equations by semi-analytical method, which known as NHPM, introduced by Biazar and Eslami (2011). NHPM was a new modification of Homotopy Perturbation Method (HPM), which was investigated by He (1999) and successfully implemented to discover the several kinds of boundary value problems $(\mathrm{He}$, 2006). Kumar and Singh (2009) worked on the Cole-Hopf transformation method for the solutions of nonlinear reaction diffusion equation. Kumar and Singh (2010) used HPM to develop 
International Journal of Mathematical, Engineering and Management Sciences

Vol. 4, No. 6, 1483-1495, 2019

https://dx.doi.org/10.33889/IJMEMS.2019.4.6-117

the solution of reaction-diffusion equations and solve the Kolmogorov-Petrovsly-Piskunov equations. Singh and Kumar (2017) worked on the differential transformation method and HPM to elucidate the reaction-diffusion equations and compare with the variational iteration method (VIM) numerous illustrations are provided, and discuss the capability of each method. Again, Singh et al. (2019) extended the result of Fisher equation by HPM.

Mostly researcher has been worked on the solution of BH equations. Wang et al. (1990) worked on the solitary wave solutions of the generalized BH equation. The singular manifold method and non-classical symmetries were used for BH equation presented by Estevez (1994). In the last few years, several powerful mathematical methods such as ADM by Hashim et al. (2006), VIM Batiha et al. (2008), Spectral collocation method Darvishi et al. (2008), Differential quadrature method Murat and Gurhan (2009), Homotopy analysis method by Molabahrami and Khani (2009), Exp-function method by Gao and Zhao (2010) Haar wavelet method by Celik (2011), Hyperbolic tangent method by Krisnangkura et al. (2012), G'/G Expansion method by Zhu (2016), Hybrid B-Spline collocation method by Wasim et al. (2018). The two combined forms of Fisher, Burgers, Huxley equations solved by HAM introduced by Babolian and Saeidian (2009). Mittal and Tripathi (2015) worked on the numerical solutions of generalized BH equations by using emplacement of cubic B-splines function. While, Ayati et al. (2014) developed the solution of Schrodinger equations by NPHM. Mirzazadeh and Ayati (2016) gave the solution of a system of Burgers equations by NHPM. Aronov and Zazhigalkin (2017) developed a duopoly model which has conveyed the experimental effectiveness condition with the patent. Gad-Allah and Elzaki (2017) used the application of NHPM for solving non-linear partial differential equation. In this work we used the NHPM for the solution of generalized BH. NHPM is the first projected solution that has been used to reach the precise solution of the equation.

\section{Mathematical Formulations}

Consider a generalized nonlinear Burgers-Huxley equation

$\frac{\partial w}{\partial t}=\frac{\partial^{2} w}{\partial x^{2}}+\alpha w^{\delta} \frac{\partial w}{\partial x}+\beta w\left(1-w^{\delta}\right)\left(w^{\delta}-\gamma\right) \quad 0 \leq x \leq 1, t \geq 0$

where, $w=w(x, t)$ be the unknown function of $x$ and $t . \alpha, \beta, \delta \geq 0$ be the real constant and $\gamma$ be the parameter, $\delta$ be the positive integer.

The value $\alpha=1, \beta=0, \delta=1$ reduce the Equation (1) to the Burgers equation which has been developed Burgers model of turbulence, and measured the theory of shock waves with the different numerical method applied to study of a class of physical flow.

The value $\alpha=\beta=\delta=1$ Equation (1) reduced to the Burgers-Huxley equation.

$\frac{\partial w}{\partial t}=\frac{\partial^{2} w}{\partial x^{2}}+w \frac{\partial w}{\partial x}+w(1-w)(w-\gamma)$

With the help of NHPM, solving the Equation (2) for real axis $-\infty<x<\infty$ with primary conditions.

$w(x, 0)=f(x), \quad-\infty<x<\infty$ 
International Journal of Mathematical, Engineering and Management Sciences

Vol. 4, No. 6, 1483-1495, 2019

https://dx.doi.org/10.33889/IJMEMS.2019.4.6-117

\section{Basic Idea of New Homotopy Perturbation Method for Burgers-Huxley Equation}

We consider BH equation in the following form in this segment

$\frac{\partial w}{\partial t}=\frac{\partial^{2} w}{\partial x^{2}}+w \frac{\partial w}{\partial x}+w(1-w)(w-\gamma)$

Subject to the initial conditions

$w(x, 0)=f(x), \quad-\infty<x<\infty$

To solve Eq. (4) with the conditions by NHPM, we developed a homotopy as follows:

$(1-p)\left(\frac{\partial w}{\partial t}(x, t)-w_{0}(x, t)\right)+p\left(\frac{\partial w}{\partial t}-\frac{\partial^{2} w}{\partial x^{2}}-w \frac{\partial w}{\partial x}-w(1-w)(w-\gamma)\right)=0$

or

$\frac{\partial w}{\partial t}(x, t)=w_{0}(x, t)-p\left(w_{0}(x, t)-\frac{\partial^{2} w}{\partial x^{2}}-w \frac{\partial w}{\partial x}-w(1-w)(w-\gamma)\right)$

By implementing the inverse operator, $L^{-1}=\int_{t_{0}}^{t}() d$.$t , both sides of the Equation (7), we get$

$w(x, t)=w(x, 0)+\int_{0}^{t}\left(w_{0}(x, t)\right) d t-p \int_{0}^{t}\left(w_{0}(x, t)-\frac{\partial^{2} w}{\partial x^{2}}-w \frac{\partial w}{\partial x}-w(1-w)(w-\gamma)\right) d t$

Presume the solution of Equation (8) in the given form:

$w=W_{0}+p W_{1}+p^{2} W_{2}+p^{3} W_{3}+\cdots$

To replace the Equation (9) in the Equation (8) and compare the rank of equal power of p, it is as follows

$$
\begin{aligned}
& p^{0}: W_{0}(x, t)=w(x, 0)+\int_{0}^{t}\left(w_{0}(x, t)\right) d t \\
& p^{1}: W_{1}(x, t)=-\int_{0}^{t}\left(\begin{array}{c}
\left.w_{0}(x, t)-\frac{\partial^{2} W_{0}}{\partial x^{2}}-W_{0} \frac{\partial W_{0}}{\partial x}-W_{0}\left(1-W_{0}\right)\left(W_{0}-\gamma\right)\right) d t \\
p^{2}: W_{2}(x, t)=\int_{0}^{t}\left(\begin{array}{c}
\frac{\partial^{2} W_{1}}{\partial x^{2}}+W_{1} \frac{\partial W_{0}}{\partial x}+W_{0} \frac{\partial W_{1}}{\partial x}+W_{1}\left(1-W_{0}\right)\left(W_{0}-\gamma\right) \\
+W_{0}\left(1-W_{0}\right) W_{1}-W_{0} W_{1}\left(W_{0}-\gamma\right)
\end{array}\right) d t \\
p^{3}: W_{3}(x, t)=\int_{0}^{t}\left(\begin{array}{c}
\frac{\partial^{2} W_{2}}{\partial x^{2}}+W_{2} \frac{\partial W_{0}}{\partial x}+W_{1} \frac{\partial W_{1}}{\partial x}+W_{0} \frac{\partial W_{2}}{\partial x}+W_{1}\left(\left(1-W_{0}\right) W_{1}-W_{1}\left(W_{0}-\gamma\right)\right) \\
\left.+W_{0}\left(1-W_{0}\right) W_{2}-W_{1} W_{1}-W_{2}\left(W_{0}-\gamma\right)\right)+W_{2}\left(1-W_{0}\right)\left(W_{0}-\gamma\right)
\end{array}\right) d t
\end{array}\right.
\end{aligned}
$$

Similarly others. 
International Journal of Mathematical, Engineering and Management Sciences

Vol. 4, No. 6, 1483-1495, 2019

https://dx.doi.org/10.33889/IJMEMS.2019.4.6-117

Assume $w_{0}(x, t)=\sum_{i=0}^{\infty} a_{i}(x) P_{i}(t), P_{i}(t)=t^{i}$

where $a_{0}(x), a_{1}(x), a_{2}(x), a_{3}(x), \ldots$ are unknown coefficients, and $P_{0}(t), P_{1}(t), P_{2}(t)$, $P_{3}(t), \ldots$ are specified functions depending on the problem.

Now if we solve these Equations (10) to (13) in such a way that consider the hypothesis, $W_{1}(x, t)=0$ then, the Equations (10)-(13) yield to

$$
W_{j}(x, t)=0, \quad j=2,3,4, \ldots . .
$$

So, the solution of the Equation (4) can be given in the form

$w(x, t)=W_{0}(x, t)=f(x)+\sum_{i=0}^{\infty} a_{i}(x) \frac{P_{i+1}(t)}{i+1}$

where, $P_{i}(t)=t^{i}, a_{i}(x) i=0,1,2,3, \ldots .$. , are unknown coefficients that should be calculated.

\section{Numerical Illustration and Discussion}

In this segment, we apply the NHPM depicted previously past segment, for the numerical result of the proposed model describing the analytical approximation of the equation of the $\mathrm{BH}$ equation of reaction diffusion equation. The given case study of the estimated solution received from $\mathrm{BH}$ equations is similar to the precise solution and is to check the efficiency and accuracy of the system.

Example 1 Solve the following Burgers-Huxley equation taking $\gamma=1$ in Equation (4) Babolian and Saeidian (2009).

$\frac{\partial w}{\partial t}=\frac{\partial^{2} w}{\partial x^{2}}+w \frac{\partial w}{\partial x}+w(1-w)(w-1)$

Subject to the initial conditions

$w(x, 0)=\frac{e^{-\frac{x}{4}}}{e^{\frac{x}{4}}+e^{-\frac{x}{4}}}$,

To solve equation (16) with the conditions by NHPM, we developed a homotopy as follows:

$(1-p)\left(\frac{\partial w}{\partial t}(x, t)-w_{0}(x, t)\right)+p\left(\frac{\partial w}{\partial t}-\frac{\partial^{2} w}{\partial x^{2}}-w \frac{\partial w}{\partial x}-w(1-w)(w-1)\right)=0$

$\frac{\partial w}{\partial t}(x, t)=w_{0}(x, t)-p\left(w_{0}(x, t)-\frac{\partial^{2} w}{\partial x^{2}}-w \frac{\partial w}{\partial x}-w(1-w)(w-1)\right)$

By implementing the inverse operator, $L^{-1}=\int_{t_{0}}^{t}() d$.$t , both sides of the equation (19), we get$

$w(x, t)=w(x, 0)+\int_{0}^{t}\left(w_{0}(x, t)\right) d t-p \int_{0}^{t}\left(w_{0}(x, t)-\frac{\partial^{2} w}{\partial x^{2}}-w \frac{\partial w}{\partial x}-w(1-w)(w-1)\right) d t$ 
International Journal of Mathematical, Engineering and Management Sciences

Vol. 4, No. 6, 1483-1495, 2019

https://dx.doi.org/10.33889/IJMEMS.2019.4.6-117

Presume the solution of equation (20) in the given form:

$w=W_{0}+p W_{1}+p^{2} W_{2}+p^{3} W_{3}+\cdots$

To replace the equation (21) in the equation (20) and compare the rank of equal power of $p$, it is as follows

$$
\begin{aligned}
& p^{0}: W_{0}(x, t)=w(x, 0)+\int_{0}^{t}\left(w_{0}(x, t)\right) d t \\
& p^{1}: W_{1}(x, t)=-\int_{0}^{t}\left(w_{0}(x, t)-\frac{\partial^{2} W_{0}}{\partial x^{2}}-W_{0} \frac{\partial W_{0}}{\partial x}-W_{0}\left(1-W_{0}\right)\left(W_{0}-1\right)\right) d t \\
& p^{2}: W_{2}(x, t)=\int_{0}^{t}\left(\begin{array}{c}
\frac{\partial^{2} W_{1}}{\partial x^{2}}+W_{1} \frac{\partial W_{0}}{\partial x}+W_{0} \frac{\partial W_{1}}{\partial x}+W_{1}\left(1-W_{0}\right)\left(W_{0}-1\right) \\
+W_{0}\left(1-W_{0}\right) W_{1}-W_{0} W_{1}\left(W_{0}-1\right)
\end{array}\right) d t \\
& p^{3}: W_{3}(x, t)=\int_{0}^{t}\left(\begin{array}{c}
\frac{\partial^{2} W_{2}}{\partial x^{2}}+W_{2} \frac{\partial W_{0}}{\partial x}+W_{1} \frac{\partial W_{1}}{\partial x}+W_{0} \frac{\partial W_{2}}{\partial x}+W_{1}\left(\left(1-W_{0}\right) W_{1}-W_{1}\left(W_{0}-1\right)\right) \\
+W_{0}\left(\left(1-W_{0}\right) W_{2}-W_{1} W_{1}-W_{2}\left(W_{0}-1\right)\right)+W_{2}\left(1-W_{0}\right)\left(W_{0}-1\right)
\end{array}\right) d t
\end{aligned}
$$

Similarly, others.

Assume $w_{0}(x, t)=\sum_{i=0}^{\infty} a_{i}(x) P_{i}(t), P_{i}(t)=t^{i}$

Now if we solve these Equations (22) to (25) leads the following results

$W_{0}(x, t)=\frac{e^{-\frac{x}{4}}}{e^{\frac{x}{4}}+e^{-\frac{x}{4}}}+\sum_{i=0}^{\infty} a_{i}(x) \frac{t^{i+1}}{i+1}$

Use the value of $W_{0}(x, t)$ in equation (23), and $W_{1}(x, t)$ vanish, then calculate the coefficient of $a_{i}(x) i=0,1,2,3, \ldots .$. using MATLAB software will be obtained as follows:

$a_{0}=-\frac{3}{4\left(e^{\frac{x}{4}}+e^{-\frac{x}{4}}\right)^{2}}, \quad a_{1}=\frac{9\left(e^{\frac{x}{4}}-e^{-\frac{x}{4}}\right)}{16\left(e^{\frac{x}{4}}+e^{-\frac{x}{4}}\right)^{3}}, \quad a_{2}=-\frac{27\left(\left(e^{\frac{x}{4}}\right)^{2}+\left(e^{-\frac{x}{4}}\right)^{2}-4\right)}{128\left(e^{\frac{x}{4}}+e^{-\frac{x}{4}}\right)^{4}}, \ldots \ldots$

Similarly, others.

Therefore, the obtained as the following form:

$$
\begin{aligned}
& w(x, t)=W_{0}(x, t)=\frac{e^{-\frac{x}{4}}}{e^{\frac{x}{4}}+e^{-\frac{x}{4}}}-\frac{3}{4\left(e^{\frac{x}{4}}+e^{-\frac{x}{4}}\right)^{2}} t+\frac{9\left(e^{\frac{x}{4}}-e^{-\frac{x}{4}}\right)}{16\left(e^{\frac{x}{4}}+e^{-\frac{x}{4}}\right)^{3}} \frac{t^{2}}{2} \\
& -\frac{27\left(\left(e^{\frac{x}{4}}\right)^{2}+\left(e^{-\frac{x}{4}}\right)^{2}-4\right)}{128\left(e^{\frac{x}{4}}+e^{-\frac{x}{4}}\right)^{4}} \frac{t^{3}}{3}+\cdots
\end{aligned}
$$


International Journal of Mathematical, Engineering and Management Sciences

Vol. 4, No. 6, 1483-1495, 2019

https://dx.doi.org/10.33889/IJMEMS.2019.4.6-117

The expansion of $\frac{1}{2}-\frac{1}{2} \tanh \left(\frac{x}{4}+\frac{3 t}{8}\right)=\frac{1}{2}-\frac{1}{2} \frac{e^{\left(\frac{x}{4}+\frac{3 t}{8}\right)}-e^{-\left(\frac{x}{4}+\frac{3 t}{8}\right)}}{e^{\left(\frac{x}{4}+\frac{3 t}{8}\right)}+e^{-\left(\frac{x}{4}+\frac{3 t}{8}\right)}}$ Taylor series is given below

$$
\begin{aligned}
& \frac{1}{2}-\frac{1}{2} \frac{e^{\left(\frac{x}{4}+\frac{3 t}{8}\right)}-e^{-\left(\frac{x}{4}+\frac{3 t}{8}\right)}}{e^{\left(\frac{x}{4}+\frac{3 t}{8}\right)}+e^{-\left(\frac{x}{4}+\frac{3 t}{8}\right)}}=\frac{1}{2}-\frac{1}{2} \tanh \left(\frac{x}{4}+\frac{3 t}{8}\right) \\
& =\frac{e^{-\frac{x}{4}}}{e^{\frac{x}{4}}+e^{-\frac{x}{4}}}-\frac{3}{4\left(e^{\frac{x}{4}}+e^{-\frac{x}{4}}\right)^{2}} t+\frac{9\left(e^{\frac{x}{4}}-e^{-\frac{x}{4}}\right)}{16\left(e^{\frac{x}{4}}+e^{-\frac{x}{4}}\right)^{3}} \frac{t^{2}}{2}-\frac{27\left(\left(e^{\frac{x}{4}}\right)^{2}+\left(e^{-\frac{x}{4}}\right)^{2}-4\right)}{128\left(e^{\frac{x}{4}}+e^{-\frac{x}{4}}\right)^{4}} \frac{t^{3}}{3}+\cdots
\end{aligned}
$$

This is the same as Taylor series expansion of the precise solution. By substituting equation (29) into equation (28), equation (28) can be reduced to:

$$
w(x, t)=\frac{1}{2}-\frac{1}{2} \frac{e^{\left(\frac{x}{4}+\frac{3 t}{8}\right)}-e^{-\left(\frac{x}{4}+\frac{3 t}{8}\right)}}{e^{\left(\frac{x}{4}+\frac{3 t}{8}\right)}+e^{-\left(\frac{x}{4}+\frac{3 t}{8}\right)}}=\frac{1}{2}-\frac{1}{2} \tanh \left(\frac{x}{4}+\frac{3 t}{8}\right)
$$

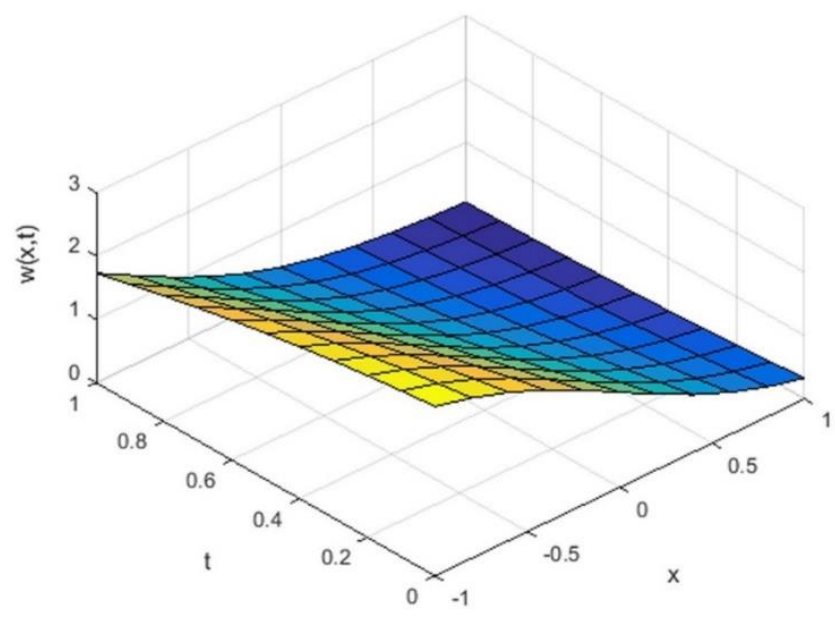

Figure 1. 3-D demonstration for the result of the example 1

Figure 1 display the 3-D demonstration for the result of the example 1 of equation (16) by NHPM with the use of MATLAB takes $t=0$ to $t=1$ and $x=-1$ to $x=1$. Whereas the figure 2 and figure 3 represent the two dimensional representation of the solution equation (16) taking the value of $t=0, t=0.5$ and $t=1$ and $x=-1$ to $x=1$. The value of $x$ increases the value of $w(x, t)$ is decrease for all value of $t=0, t=0.5$ and $t=1$ in figure 2 The figure 3 display the 2$\mathrm{D}$ demonstration for the result of equation (16) taking the value of $x=-1, x=-0.5, x=$ $0, x=0.5$ and $x=1$ and $t=0$ to $t=1$. The value of $t$ increases, then the value of $w(x, t)$ decreases for all value of $x$. The equation (30) represents the precise solution of BH equation (16) by NHPM. 
International Journal of Mathematical, Engineering and Management Sciences

Vol. 4, No. 6, 1483-1495, 2019

https://dx.doi.org/10.33889/IJMEMS.2019.4.6-117

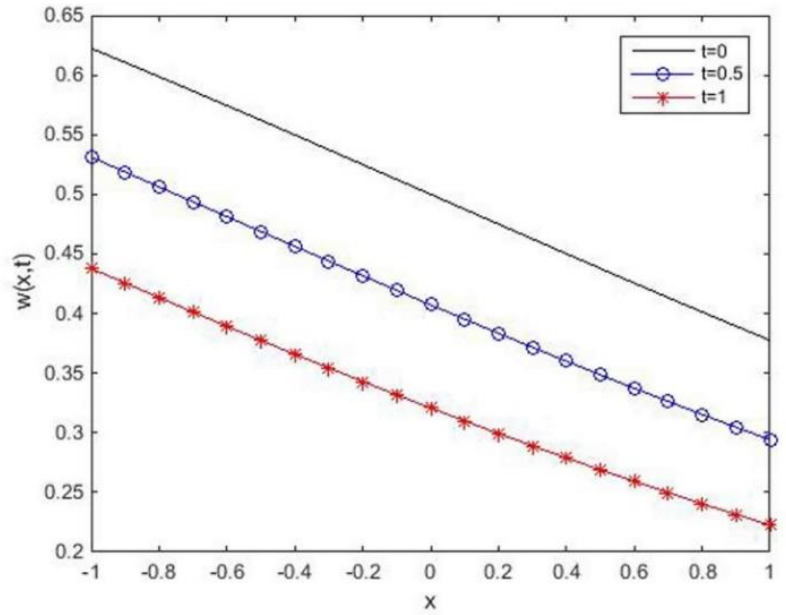

Figure 2. 2-D demonstration for the result of equation (16) by taking $t=0, t=0.5$ and $t=1$

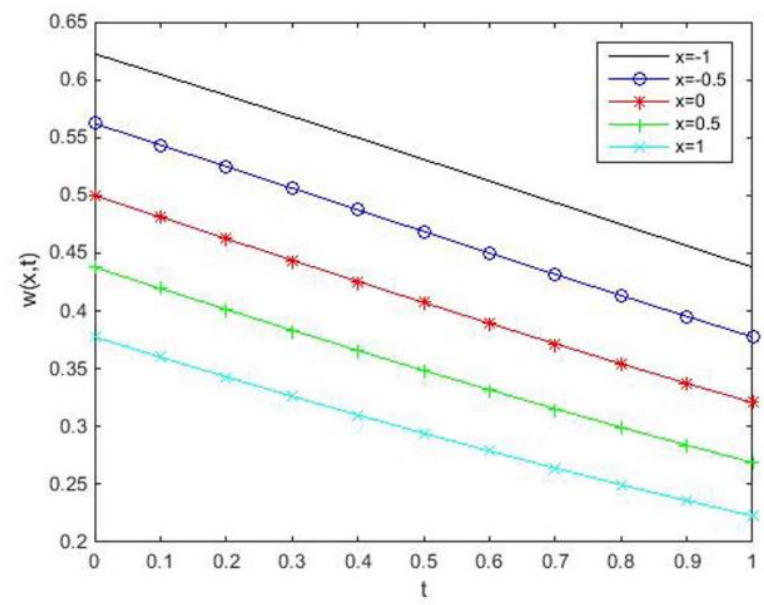

Figure 3. 2-D demonstration for the result of Equation (16) by taking $x=-1, x=-0.5, x=0, x=0.5$ and $x=1$

Example 2 Solve the following Burgers-Huxley equation taking $\alpha=2, \beta=\delta=1$ and $\gamma=3$ in Equation (1)

$\frac{\partial w}{\partial t}=\frac{\partial^{2} w}{\partial x^{2}}+2 w \frac{\partial w}{\partial x}+w(1-w)(w-3)$

Subject to the initial conditions

$$
w(x, 0)=\frac{e^{-\frac{3(\sqrt{3}-1) x}{4}}}{e^{\frac{3(\sqrt{3}-1) x}{4}}+e^{-\frac{3(\sqrt{3}-1) x}{4}}}
$$


International Journal of Mathematical, Engineering and Management Sciences

Vol. 4, No. 6, 1483-1495, 2019

https://dx.doi.org/10.33889/IJMEMS.2019.4.6-117

To solve Equation (16) with the conditions by NHPM, we developed a homotopy as follows:

$(1-p)\left(\frac{\partial w}{\partial t}(x, t)-w_{0}(x, t)\right)+p\left(\frac{\partial w}{\partial t}-\frac{\partial^{2} w}{\partial x^{2}}-2 w \frac{\partial w}{\partial x}-w(1-w)(w-3)\right)=0$

or

$\frac{\partial w}{\partial t}(x, t)=w_{0}(x, t)-p\left(w_{0}(x, t)-\frac{\partial^{2} w}{\partial x^{2}}-2 w \frac{\partial w}{\partial x}-w(1-w)(w-3)\right)$

Applying the inverse operator, $L^{-1}=\int_{t_{0}}^{t}() d$.$t , to both sides of Equation (19), we obtain$

$w(x, t)=w(x, 0)+\int_{0}^{t}\left(w_{0}(x, t)\right) d t-p \int_{0}^{t}\left(w_{0}(x, t)-\frac{\partial^{2} w}{\partial x^{2}}-2 w \frac{\partial w}{\partial x}-w(1-w)(w-3)\right) d t$

Presume the solution of Equation (35) in the given form:

$w=W_{0}+p W_{1}+p^{2} W_{2}+p^{3} W_{3}+\cdots$

Substituting Equation (36) into Equation (35) and comparing the term of equal power of $p$, it follows that-

$p^{0}: W_{0}(x, t)=w(x, 0)+\int_{0}^{t}\left(w_{0}(x, t)\right) d t$

$p^{1}: W_{1}(x, t)=-\int_{0}^{t}\left(w_{0}(x, t)-\frac{\partial^{2} W_{0}}{\partial x^{2}}-2 W_{0} \frac{\partial W_{0}}{\partial x}-W_{0}\left(1-W_{0}\right)\left(W_{0}-1\right)\right) d t$

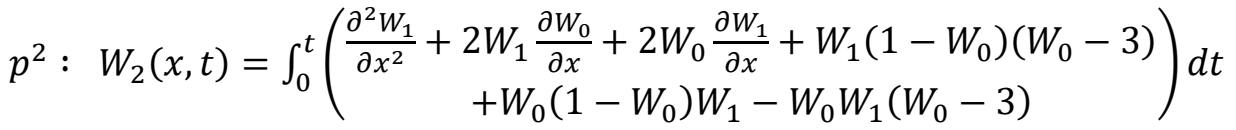

$p^{3}: W_{3}(x, t)=$

$\int_{0}^{t}\left(\begin{array}{c}\frac{\partial^{2} W_{2}}{\partial x^{2}}+2 W_{2} \frac{\partial W_{0}}{\partial x}+2 W_{1} \frac{\partial W_{1}}{\partial x}+2 W_{0} \frac{\partial W_{2}}{\partial x}+W_{1}\left(\left(1-W_{0}\right) W_{1}-W_{1}\left(W_{0}-3\right)\right) \\ +W_{0}\left(\left(1-W_{0}\right) W_{2}-W_{1} W_{1}-W_{2}\left(W_{0}-3\right)\right)+W_{2}\left(1-W_{0}\right)\left(W_{0}-3\right)\end{array}\right) d t$

Similarly, others.

Assume $w_{0}(x, t)=\sum_{i=0}^{\infty} a_{i}(x) P_{i}(t), P_{i}(t)=t^{i}$

Now if we solve these Equations (37) to (40) leads the following results

$W_{0}(x, t)=\frac{3 e^{-\frac{3(\sqrt{3}-1) x}{4}}}{e^{\frac{3(\sqrt{3}-1) x}{4}}+e^{-\frac{3(\sqrt{3}-1) x}{4}}}+\sum_{i=0}^{\infty} a_{i}(x) \frac{t^{i+1}}{i+1}$

Use the value of $W_{0}(x, t)$ in Equation (38), and $W_{1}(x, t)$ vanish, then calculate the coefficient of $a_{i}(x) i=0,1,2,3, \ldots .$. using MATLAB software will be obtained as follows: 
International Journal of Mathematical, Engineering and Management Sciences

Vol. 4, No. 6, 1483-1495, 2019

https://dx.doi.org/10.33889/IJMEMS.2019.4.6-117

$$
\begin{aligned}
& a_{0}=-\frac{9(3 \sqrt{3}-4)}{2\left(e^{\frac{3(\sqrt{3}-1) x}{4}}+e^{-\frac{3(\sqrt{3}-1) x}{4}}\right)^{2}}, a_{1}=\frac{27\left(e^{\frac{3(\sqrt{3}-1) x}{4}}-e^{\left.-\frac{3(\sqrt{3}-1) x}{4}\right)(43-24 \sqrt{3})}\right.}{8\left(e^{\frac{3(\sqrt{3}-1) x}{4}}+e^{\left.-\frac{3(\sqrt{3}-1) x}{4}\right)^{3}}\right.}
\end{aligned}
$$

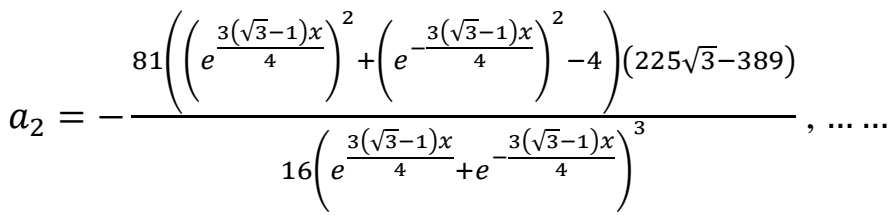

Similarly, others.

Therefore, the obtained results as the following form:

$$
\begin{aligned}
& w(x, t)=W_{0}(x, t)=\frac{3 e^{-\frac{3(\sqrt{3}-1) x}{4}}}{e^{\frac{3(\sqrt{3}-1) x}{4}}+e^{-\frac{3(\sqrt{3}-1) x}{4}}}-\frac{9(3 \sqrt{3}-4)}{2\left(e^{\frac{3(\sqrt{3}-1) x}{4}}+e^{\left.-\frac{3(\sqrt{3}-1) x}{4}\right)^{2}}\right.}+\frac{t^{2}}{2} \times
\end{aligned}
$$

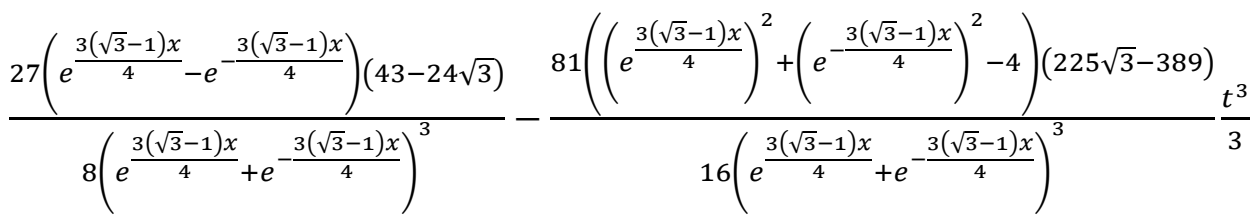

The Taylor series expansion of $\frac{3}{2}-\frac{3}{2} \frac{e^{\left(\frac{3(\sqrt{3}-1) x}{4}+\frac{3(3 \sqrt{3}-4) t}{4}\right)}-e^{-\left(\frac{3(\sqrt{3}-1) x}{4}+\frac{3(3 \sqrt{3}-4) t}{4}\right)}}{e^{\left(\frac{3(\sqrt{3}-1) x}{4}+\frac{3(3 \sqrt{3}-4) t}{4}\right)}+e^{-\left(\frac{3(\sqrt{3}-1) x}{4}+\frac{3(3 \sqrt{3}-4) t}{4}\right)}}=\frac{3}{2}-$ $\frac{3}{2} \tanh \left(\frac{3(\sqrt{3}-1) x}{4}+\frac{3(3 \sqrt{3}-4) t}{4}\right)$ can be written as

$$
\begin{aligned}
& \frac{3}{2}-\frac{3}{2} \frac{e^{\left(\frac{3(\sqrt{3}-1) x}{4}+\frac{3(3 \sqrt{3}-4) t}{4}\right)}-e^{-\left(\frac{3(\sqrt{3}-1) x}{4}+\frac{3(3 \sqrt{3}-4) t}{4}\right)}}{e^{\left(\frac{3(\sqrt{3}-1) x}{4}+\frac{3(3 \sqrt{3}-4) t}{4}\right)}+e^{-\left(\frac{3(\sqrt{3}-1) x}{4}+\frac{3(3 \sqrt{3}-4) t}{4}\right)}}=\frac{3}{2}-\frac{3}{2} \tanh \left(\frac{3(\sqrt{3}-1) x}{4}+\frac{3(3 \sqrt{3}-4) t}{4}\right)= \\
& \frac{3 e^{-\frac{3(\sqrt{3}-1) x}{4}}}{e^{\frac{3(\sqrt{3}-1) x}{4}}+e^{-\frac{3(\sqrt{3}-1) x}{4}}}-\frac{9(3 \sqrt{3}-4)}{2\left(e^{\frac{3(\sqrt{3}-1) x}{4}}+e^{\left.-\frac{3(\sqrt{3}-1) x}{4}\right)^{2}}\right.}+\frac{27\left(e^{\frac{3(\sqrt{3}-1) x}{4}}-e^{\left.-\frac{3(\sqrt{3}-1) x}{4}\right)(43-24 \sqrt{3})}\right.}{8\left(e^{\frac{3(\sqrt{3}-1) x}{4}}+e^{\left.-\frac{3(\sqrt{3}-1) x}{4}\right)^{3}}\right.}-
\end{aligned}
$$

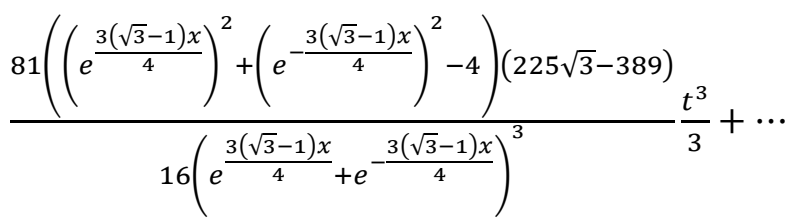

This is the same as Taylor series expansion of the precise solution. By substituting Equation (44) into Equation (43), Equation (43) can be reduced to: 
International Journal of Mathematical, Engineering and Management Sciences

Vol. 4, No. 6, 1483-1495, 2019

https://dx.doi.org/10.33889/IJMEMS.2019.4.6-117

$w(x, t)=\frac{3}{2}-\frac{3}{2} \frac{e^{\left(\frac{3(\sqrt{3}-1) x}{4}+\frac{3(3 \sqrt{3}-4) t}{4}\right)}-e^{-\left(\frac{3(\sqrt{3}-1) x}{4}+\frac{3(3 \sqrt{3}-4) t}{4}\right)}}{e^{\left(\frac{3(\sqrt{3}-1) x}{4}+\frac{3(3 \sqrt{3}-4) t}{4}\right)}+e^{-\left(\frac{3(\sqrt{3}-1) x}{4}+\frac{3(3 \sqrt{3}-4) t}{4}\right)}}=\frac{3}{2}-\frac{3}{2} \tanh \left(\frac{3(\sqrt{3}-1) x}{4}+\frac{3(3 \sqrt{3}-4) t}{4}\right)$

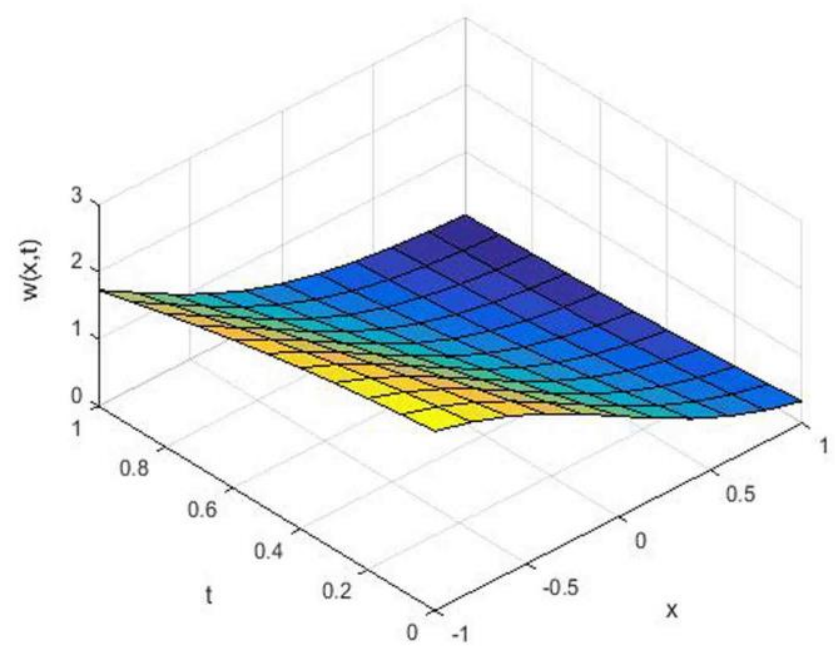

Figure 4. 3-D demonstration for the result of the example 2

Figure 4 display the 3-D demonstration for the result of the example 2 of an Equation (31) by NHPM with the use of MATLAB takes $t=0$ to $t=1$ and $x=-1$ to $x=1$.

While the figure 5 display the 2-D demonstration for the result of Equation (31) taking the value of $t=0, t=0.5$ and $t=1$ and $x=-1$ to $x=1$. The value of $x$ increases the value of $w(x, t)$ is decrease, while the figure 6 display the 2-D demonstration for the result of Equation (31) taking the value of $x=-1, x=-0.5, x=0, x=0.5$ and $x=1$ and $t=0$ to $t=1$. The value of $t$ increases the value of $w(x, t)$ is decreased for all value $x$. The Equation (45) represents the precise solution of BH Equation (31) by NHPM. 
International Journal of Mathematical, Engineering and Management Sciences

Vol. 4, No. 6, 1483-1495, 2019

https://dx.doi.org/10.33889/IJMEMS.2019.4.6-117

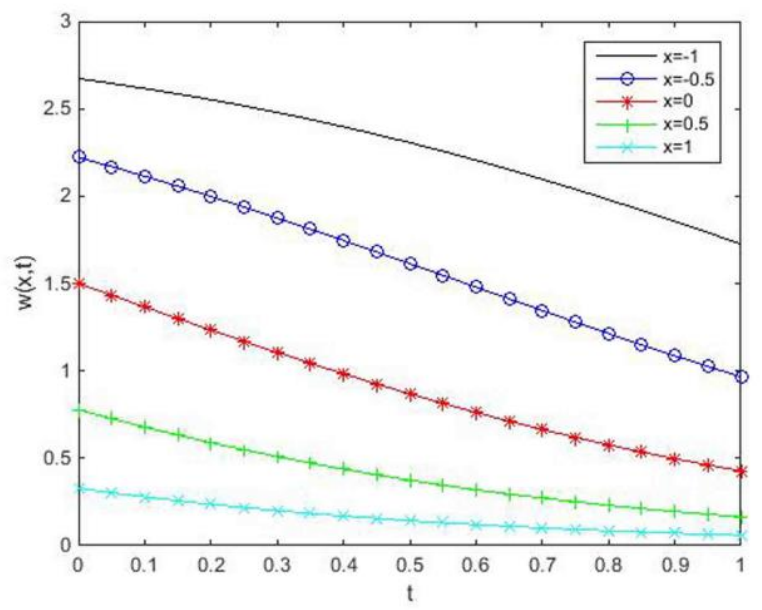

Figure 5. 2-D demonstration for the result of the example 2 by taking $t=0, t=0.5$ and $t=1$

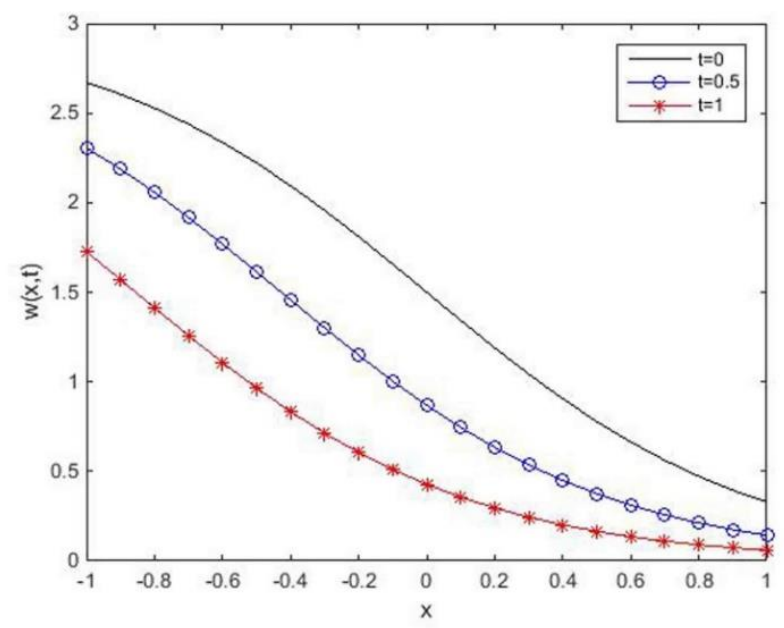

Figure 6. 2-D demonstration for the result of the example 2 by taking $x=-1, x=-0.5, x=0.5$ and $x=1$

\section{Conclusion}

The NHPM has been effectively useful to find the precise solution of the generalized $\mathrm{BH}$ equation of nonlinear reaction-diffusion equation. The consequence obtained is a good guess of the Taylor expansion of precise solutions. To examine the validity, usability and flexibility of NHPM, two case studies are provided for the precise solution of the BH equation. It displays that NHPM is an optimistic application to solving the linear and nonlinear reaction-diffusion equations. MATLAB software is used to plot the two-dimensions and three-dimensional graphical representation and compute the series solution found from the NHPM. 
International Journal of Mathematical, Engineering and Management Sciences

Vol. 4, No. 6, 1483-1495, 2019

https://dx.doi.org/10.33889/IJMEMS.2019.4.6-117

\section{Conflict of Interest}

The authors confirm that there is no conflict of interest to declare for this publication.

\section{Acknowledgments}

We are grateful to all the reviewers for their encouraging comments and valuable corrections.

\section{References}

Aronov, I., \& Zazhigalkin, A. (2017). Diffusion of innovations: patenting or standardization. International Journal of Mathematical, Engineering and Management Sciences, 2(2), 64-73.

Ayati, Z., Biazar, J., \& Ebrahimi, S. (2014). A new homotopy perturbation method for solving linear and nonlinear Schrodinger equations. Journal of Interpolation and Approximation in Scientific Computing, 2014, 1-8.

Babolian, E., \& Saeidian, J. (2009). Analytic approximate solutions to Burgers, Fisher, Huxley equations and two combined forms of these equations. Communications in Nonlinear Science and Numerical Simulation, 14(5), 1984-1992.

Batiha, B., Noorani, M.S.M., \& Hashim, I. (2008). Application of variational iteration method to the generalized Burgers-Huxley equation. Chaos, Solitons \& Fractals, 36(3), 660-663.

Biazar, J., \& Eslami, M. (2011). A new homotopy perturbation method for solving systems of partial differential equations. Computers \& Mathematics with Applications, 62(1), 225-234.

Celik, I. (2011). Haar wavelet method for solving generalized Burgers-Huxley equation. Arab Journal of Mathematical Sciences, 18(1), 25-37.

Darvishi, M.T., Kheybari, S., \& Khani, F. (2008). Spectral collocation method and Darvishi's preconditioning to solve the generalized Burgers-Huxley equation. Communications in Nonlinear Science and Numerical Simulation, 13(10), 2091-2103.

Estevez, P.G. (1994). Non-classical symmetries and the singular manifold method: the Burgers and the Burgers-Huxley equations. Journal of Physics A: Mathematical and General, 27(6), 2113-2127.

Gao, H., \& Zhao, R.X. (2010). New exact solutions to the generalized Burgers-Huxley equation. Applied Mathematics and Computation, 217(4), 1598-1603.

Gad-Allah, M.R., \& Elzaki, T.M. (2017). Application of the new Homotopy perturbation method for solving non-linear partial differential equations, Journal of Computational and Theoretical Nanoscience, 14, 1-9.

Hashim, I., Noorani, M.S.M., \& Said Al-Hadidi, M.R. (2006). Solving the generalized Burgers-Huxley equation using the Adomian decomposition method. Mathematical and Computer Modelling, 43(1112), 1404-1411.

He, J.H. (1999). Homotopy perturbation technique. Computational Methods in Applied Mechanics and Engineering, 178(3-4), 257-262.

He, J.H. (2006). Homotopy perturbation method for solving boundary value problems. Physics Letters A, $350(1-2), 87-88$.

Kumar, S., \& Singh, R. (2009). Exact and numerical solutions of nonlinear reaction diffusion equation by using the Cole-Hopf transformation. International Transactions in Mathematical Sciences and Computer, 2(2), 241-252.

Kumar, S., \& Singh, R. (2010). Solution of reaction-diffusion equations by using homotopy perturbation method. International Journal of Stability and Fluid Mechanics, 1(2), 235-244. 
International Journal of Mathematical, Engineering and Management Sciences

Vol. 4, No. 6, 1483-1495, 2019

https://dx.doi.org/10.33889/IJMEMS.2019.4.6-117

Krisnangkura, M., Chinviriyasit, S., \& Chinviriyasit, W. (2012). Analytic study of the generalized Burger's-Huxley equation by hyperbolic tangent method. Applied Mathematics and Computation, 218, 10843-10847.

Mirzazadeh, M., \& Ayati Z. (2016). New homotopy perturbation method for system of Burgers equations. Alexandria Engineering Journal, 55(2), 1619-1624.

Mittal, R.C., \& Tripathi, A. (2015). Numerical solutions of generalized Burgers-Fisher and generalized Burgers-Huxley equations using collocation of cubic B-splines. International Journal of Computer Mathematics, 92(5), 1053-1077.

Molabahrami, A., \& Khani, F. (2009). The homotopy analysis method to solve the Burgers-Huxley equation. Nonlinear Analysis: Real World Applications, 10(2), 589-600.

Murat, S., \& Gurhan, G. (2009). Numerical solutions of the generalized Burgers-Huxley equation by a differential quadrature method. Mathematical Problems in Engineering, 2009, Article ID 370765, 11 pages, 2009. https://doi.org/10.1155/2009/370765.

Satsuma, J. (1987). Exact solutions of Burgers' equation with reaction terms. In: Ablowitz, M., Fuchssteiner, B., \& Kruskal, M. (Eds) In Topics in Soliton Theory and Exactly Solvable Nonlinear Equations: Proceedings of the Conference on Nonlinear Evolution Equations, Solitons and the Inverse Scattering Transform (pp. 255-262). World Scientific, Singapore.

Singh, R., Maurya, D.K., \& Rajoria, Y.K. (2019). A novel approach of homotopy perturbation technique to solution of non-linear Fisher equation. International Journal of Applied Engineering Research, 14(4), 957-964.

Singh, R., \& Kumar A. (2017). A comparative study of Homotopy perturbation method with differential transformation method to solve a reaction diffusion equation. Indian Journal of Science and Technology, 10(30), 1-9.

Wang, X.Y., Zhu, Z.S., \& Lu, Y.K. (1990). Solitary wave solutions of the generalised Burgers-Huxley equation. Journal of Physics A: Mathematical and General, 23(3), 271-275.

Wasim, I., Abbas, M., \& Amin, M. (2018). Hybrid B-Spline collocation method for solving the generalized Burgers-Fisher and Burgers-Huxley equations. Mathematical Problems in Engineering, 2018, Article ID 6143934, 18 pages, 2018. https://doi.org/10.1155/2018/6143934.

Zhu, M. (2016). Solving the Burgers-Huxley equation by G'/G expansion method. Journal of Applied Mathematics and Physics, 4(7), 1371-1377. 